\title{
Basic Properties of an Alginate Impression Material Supplemented with Chlorhexidine I. Disinfectant Effects on Oral Microbes
}

\author{
Hajime Tanaka ${ }^{1}$, Shuji Ebara ${ }^{1}$, Akiyoshi Sugawara ${ }^{2}$, \\ Minoru Nishiyama ${ }^{2}$ and Kunio Hayashi ${ }^{1}$
}

(Received 17 January and accepted 10 February 1994)

Key words: impression material, disinfection, oral microbes, chlorhexidine

\begin{abstract}
To examine the disinfectant effects on oral microbes of an alginate impression material supplemented with $1 \%$ chlorhexidine as the aseptic ingredient (Coe Hydrophilic Gel, CHG), several cell suspensions of oral microbes ( 3 Gram-positives, 2 Gram-negatives and 1 yeast) were exposed to the surface of cast CHG or that of a control impression material for 5 or $30 \mathrm{~min}$. They were then transferred to the surfaces of agar plates. Colony formation was estimated after culture for several incubation periods. No growth of bacteria on the plates was observed when the suspensions had been exposed to $\mathrm{CHG}$ even for $5 \mathrm{~min}$, whereas for the control material, all the bacteria formed colonies. The yeast showed colony formation irrespective of the kind of impression material and the duration of exposure. Thus, it appears that use of an impression material supplemented with $1 \%$ chlorhexidine such as $\mathrm{CHG}$ may protect clinical staff and dental technicians from possible bacterial infections associated with the impression procedures.
\end{abstract}

\section{Introduction}

Recent studies have shown that most alginate powders are contaminated with microbes before they even leave the factory ${ }^{[1-5]}$.

Consequently, a demand has arisen for impression materials which contain antimicrobial agents so that they can be kept aseptic. Coe Hydrophilic Gel (CHG), which has been available on the market for a couple of years in the U.S.A. and about a dozen other countries, is supplemented with $1 \%(\mathrm{w} / \mathrm{w})$ chlorhexidine to make the powder aseptic. However, the profile of this impression material as a disinfectant against microbes has never been reported.

If an impression material contains a certain amount of chlorhexidine, it may well show disinfectant properties during the impression procedure, besides providing asepsis of the powder. In this study, we examined the disinfectant effects of $\mathrm{CHG}$ on human oral microbes.

Strains and Preparation of Cell Suspensions

\section{Materials and Methods}

Streptococcus mitis ATCC 9811, Actinomyces naeslundii WVU 398 A and Staphylococcus aureus 209 p were chosen as Gram-positives. As Gram-negatives, Veillonella parvula ATCC 17745 and Porphyromonas gingivalis 381 were chosen. A strain of Candida albicans named Yazaki isolated from a dental student was also employed. S. mitis and A. naeslundii were incubated in brain-heart infusion (BHI) broth (Difco, U.S.A.) until early logarithmic phase, washed twice in sterile physiological saline (PS) by centrifugation, and resuspended in PS to adjust the turbidity to OD $550 \mathrm{~nm}=0.1$ after dispersal by forceful pas-

田中 -1 ${ }^{1}$, 江原修司 ${ }^{1}$, 菅原明喜 ${ }^{2}$, 西山 實 ${ }^{2}$, 林 邦雄 $^{1}$

1 Department of Bacteriology, Nihon University School of Dentistry

2 Department of Dental Materials, Nihon University School of Dentistry

To whom all correspondence should be addressed: Dr. Hajime Tanaka, Department of Bacteriology, Nihon University School of Dentistry, 1-8-13 Kanda-Surugadai, Chiyoda-ku, Tokyo 101, JAPAN 
sage through a needle $(25 \mathrm{G} \times 1$ ", Terumo, Japan) 10 times. Likewise, the other strains, $S$. aureus cultured in nutrient broth, $V$. parvula cultured in BHI broth supplemented with $1 \%$ sodium lactate, $P$. gingivalis cultured in Gifu anaerobic medium (GAM, Nissui Pharmaceutical, Japan) broth and C. albicans cultured in Sabouraud's glucose (SG) broth were respectively converted to cell suspensions with the same turbidity as that of S. mitis.

Impression Materials and Preparation of Block Specimens

Within 5 min, powders of 2 alginate impression materials, CHG (Lot No. 051193 A, GC America, U.S.A.) and Algiace II (AL II, Lot No. 237-476, Sankin, Japan) as the control materials were mixed in several standard proportions with distilled water for $30 \mathrm{~s}$, poured into Petri dishes $(\varnothing=5 \mathrm{~cm}$, Iwaki, Japan $)$ with flat lids so that the prepared blocks had a flat and smooth surface on which microbial cell suspensions could be applied and allowed to solidify.

\section{Agar Plates}

Agar plates each containing $15 \mathrm{ml}$ of medium on whose surface colony formation could be estimated were prepared in Petri dishes ( $\varnothing=10 \mathrm{~cm}$, Iwaki, Japan) for the following combinations of microbes: Mitis Salivarius Agar (Difco, U.S.A.) for S. mitis, BHI Agar supplemented with 20 ppm cadmium sulfate for $A$. naeslundii, Mannitol Salt Agar for S. aureus, Veillonella Agar for V. parvula, BHI Agar supplemented with 5\% defibrinated horse blood for P. gingivalis and SG Agar for C. albicans.

\section{Inhibition Test}

The blocks, whose surfaces were covered with $50 \mu 1$ of a microbial cell suspension, were kept in a wet box for either 5 or $30 \mathrm{~min}$ at around $23^{\circ} \mathrm{C}$. Then their surfaces were welded to those of the agar plates for $60 \mathrm{~s}$. As soon as the block was separated from an agar plate, the plate was incubated at $37^{\circ} \mathrm{C}$ for between 24 and $72 \mathrm{~h}$ under either aerobic or anaerobic conditions. After the incubation, colony formation on the plates was estimated.

\section{Results}

The colony formation on the plates is shown in Tables 1 and 2. When the cell suspensions were exposed to the surface of the blocks made of $\mathrm{CHG}$ for $5 \mathrm{~min}$, only $C$. albicans developed colonies on the plates, whereas all the microbes tested grew on the plates when placed in contact with the surface of blocks made of AL II. An identical phenomenon was observed in the case of 30-min exposure. In positive controls where the suspensions held under aerobic conditions until the end of the experiments, then directly inoculated onto the plates, all the bacteria grew. In contrast, in negative controls where the blocks without cell suspensions were welded onto the plates, no colony was detected.

Table 1 Colony formation on agar plates when cell suspensions were exposed to $\mathrm{CHG}(\mathrm{n}=6)^{*}$

\begin{tabular}{lcc}
\hline & $5 \mathrm{~min}$ & $30 \mathrm{~min}$ \\
\hline S. mitis & - & - \\
A. naeslundii & - & - \\
S. aureus & - & - \\
V.parvula & - & - \\
P. gingivalis & - & - \\
C. albicans & + & + \\
\hline
\end{tabular}

*: Each datum was obtained without any inconsistency from 3 experiments with duplicated impression blocks.

+ : Colony formation was detected on the plates.

-: Colony formation was not detected on the plates. 
Table 2 Colony formation on agar plates when cell suspensions were exposed to AL II $(n=6)^{*}$

\begin{tabular}{lcc}
\hline & $5 \mathrm{~min}$ & $30 \mathrm{~min}$ \\
\hline S. mitis & + & + \\
A. naeslundii & + & + \\
S. aureus & + & + \\
V. parvula & + & + \\
$P$. gingivalis & + & + \\
C. albicans & + & + \\
*: Each datum was obtained without any inconsistency from 3 experi- & \\
ments with duplicated impression blocks. &
\end{tabular}

\section{Discussion}

Chlorhexidine, as well as benzalkonium chloride and povidon iodine, has been reported to show high toxicity at concentrations in media $(\mathrm{w} / \mathrm{v})$ of less than $0.01 \%$ on nonsporulating bacteria or vegetative forms of sporulating bacteria, of less than $0.02 \%$ on yeasts and fungi, and of less than $0.12 \%$ on some viruses ${ }^{[6-11]}$. The concentration of chlorhexidine in a mixture of $10 \mathrm{~g}$ of CHG powder and $25 \mathrm{ml}$ of tap-water is calculated to be approximately $0.4 \%(\mathrm{w} / \mathrm{v})$.

The strains tested here were $S$. mitis, A. naeslundii and $S$. aureus as facultatively anaerobic Grampositives, $V$. parvula and $P$. gingivalis as obligately anaerobic Gram-negatives, and the yeast, $C$. albicans. These constitute the human microflora on the oral mucosa and in saliva, and tend to contaminate the surface of impressions in the mouth. Some of them are pathogens related to oral infections such as aphtha, gingivitis, periodontitis, or abscess and phlegmon. Since oral microbes never possess intracellular spores, it was expected that absolute disinfectant effects on the strains tested would be obtained.

All the bacteria tested showed total inhibition of growth on the plates, irrespective of their Gram-staining properties, by contact with the surface of the CHG blocks for only $5 \mathrm{~min}$, which would be enough time for the setting of alginate impressions in the mouth. However, the growth of the yeast was not inhibited even when exposed to $\mathrm{CHG}$ for $30 \mathrm{~min}$, which is considered to be sufficient time for dental technicians to remove a cast stone from an impression.

This result for C. albicans indicates that the concentration of chlorhexidine in water saturated at the surface of blocks made of $\mathrm{CHG}$ is not enough to inhibit the growth of yeast even after 30-min exposure. In this study, CHG may have already become solidified when the cell suspensions were applied, and thus there was very little free water at the surface of the blocks. If so, $\mathrm{CHG}$ would not be expected to disinfect viruses either. At the same time, however, it can also be speculated that solidifying CHG during setting time might produce more marked inhibitory effects on microbes, because the water exchange rate might be higher at the surface of the impression mixture adjacent to the oral mucosa, resulting in higher concentrations of chlorhexidine there. This indicates that further study on the disinfectant effects of the solidifying surface of CHG is warranted.

\section{Conclusion}

The use of an impression material supplemented with $1 \%$ chlorhexidine, such as Coe Hydrophilic Gel, may protect clinical staff and dental technicians from at least some bacterial infections associated with impression procedures.

\section{References}

[1] Rice, C. D., Moghadam, B., Gier, R. E. and Cobb, C. M.: Aerobic bacterial contamination in dental materials, Oral Surg. Oral Med. Oral Pathol., 70, 537-539, 1990

[2] Rice, C. D., Dykstra, M. A. and Gier, R. E.: Microbial contamination in four brands of irreversible 
hydrocolloid impression material, J. Prosthet. Dent., 65, 419-423, 1991

[3] Rice, C. D., Dykstra, M. A., Gier, R. E. and Cobb, C. M.: Bacterial contamination in reversible hydrocolloid impression material and gingival cord, J. Prosthet. Dent., 65, 496-499, 1991

[4] Rice, C. D., Dykstra, M. A. and Feil, P. H.: Microbial contamination in two antimicrobial and four control brands of alginate impression material, J. Prosthet. Dent., 67, 535-540, 1992

[5] Rice, C. D., Barker, B. F., Kestenbaum, T., Dykstra, M. A. and Lumpkin, D.: Intraoral vesicles occurring after alginate impressions, Oral Surg. Oral Med. Oral Pathol., 74, 698-704, 1992

[6] Ohmura, M. and Ikeda, K.: Chlorhexidine. In Disinfectants; Basic Knowledge and Proper Usage, Revised Ed., 227-235, Takasugi, M., ed., Iyaku Journal Co., Japan, 1990 (in Japanese)

[7] Shen, D. T., Leendertsen, L. W. and Gorham, J. R.: Evaluation of chemical disinfectants for Aleutian disease virus of mink A, J. Vet. Res., 42, 838-840, 1981

[8] Hudson, A. D.: Herpes simplex virus and CPR training manikins; Reducing the risk of cross-infection, Ann. Emerg. Med., 13, 1108-1110, 1984

[9] Rodgers, F. G., Hufton, P., Kurzawska, E., Molloy, C. and Morgan, S.: Morphological response of human rotavirus to ultra-violet radiation, heat and disinfectants, J. Med. Microbiol., 20, 123-130, 1985

[10] Park, J. B. and Park, N.: Effect of chlorhexidine on the in vitro and in vivo herpes simplex virus infection, Oral Surg. Oral Med. Oral Pathol., 67, 149-153, 1989

[11] Bernstein, D., Schiff, G., Echler, G., Prince, A., Feller, M. and Briner, W.: In vitro virucidal effectiveness of a $0.12 \%$-chlorhexidine gluconate mouthrinse, J. Dent. Res., 69, 874-876, 1990 